\title{
ELECTRON RADIATION EFFECTS ON CANDIDATE SOLAR SAIL MATERIAL
}

David L. Edwards ${ }^{(1)}$, William A. Hollerman ${ }^{(2)}$, Whitney S. Hubbs ${ }^{(3)}$, Perry A. Gray ${ }^{(4)}$

George E. Wertz ${ }^{(5)}$ David T. Hoppe ${ }^{(6)}$ Mary K.Nehls $^{(7)}$ Charles L. Semmel $^{(8)}$,

(1)NASAMMSFC, ED31, MSFC, AL 35812, USA, David.LEdwards@NASA.gov

(2)Dept. of Physics, University of Louisiana at Lafayette, PO Box 44210, Lafayette, LO, 70504, USA

(3)NASA/MSFC,ED31,MSFC,AL 35812,USA, Whitney.S.Hubbs@NASA.gov

(4)ICRC,ED31,MSFC,AL 35812, USA Perry.A.Gray@NASA.gov

(5)NASA/MSFC, ED31, MSFC,AL 35812, George.E.Wertz@NASA.gov

(6)NASAMSFC, ED31,MSFC,AL35812, USA David.T.Hoppe@NASA.gov

(7)NASAMSFC, ED31 MSFC,AL 35812, USA Marv.K.Nehls@NASA.gov

(8) QUALIS, ED31, MSFC, AL 35812, USA Charles.L.Semmel@NASA.gov

\begin{abstract}
Solar sailing is a unique form of propulsion where a spacecraft gains momentum from incident photons. Solar sails are not limited by reaction mass and provide continual acceleration, reduced only by the lifetime of the lightweight film in the space environment and the distance to the Sun. Once thought to be difficult or impossible, solar sailing has come out of science fiction and into the realm of possibility. Any spacecraft using this propulsion method would need to deploy a thin sail that could be as large as many kilometers in extent. The availability of strong, ultra lightweight, and radiation resistant materials will determine the future of solar sailing.
\end{abstract}

The National Aeronautics and Space Administration's (NASA) Marshall Space Flight Center (MSFC) is concentrating research into the utilization of ultra lightweight materials for spacecraft propulsion. The Space Environmental Effects Team at MSFC is actively characterizing candidate solar sail material to evaluate the thermo-optical and mechanical properties after exposure to space environmental effects. This paper will describe the irradiation of candidate solar sail materials to energetic electrons, in vacuum, to determine the hardness of several candidate sail materials. 
Hardness in this context is defined as the amount of electron fluence (electrons/area) required to cause the sail material to fail. This paper will describe the testing procedures and preliminary results of this investigation.

\section{INTRODUCTION}

Solar sailing has become an increasingly attractive propulsion system for long term missions where the cost of chemically propelled vehicles would be prohibitive. Solar sails would allow applications such as station keeping in areas that cannot be occupied by conventionally propelled satellites due to excessive fuel requirements. Solar sail powered missions are capable of achieving non-Keplerian orbits and can provide high velocities for long duration missions. Conventional propulsion techniques require the acceleration of mass expelled from the spacecraft to produce thrust. This mass is finite and will limit the available velocity change of the spacecraft. Solar sails require virtually no fuel mass, which make them attractive for in-space propulsion.

Functional solar sails must be resistant to the effects of space environmental exposure. Previous investigations have indicated that the thermo-optical properties of candidate solar sail materials are not significantly degraded by space exposure [1]. This paper investigates the effect of radiation exposure on the mechanical properties of candidate sail material. Sails, when fully deployed, can have biaxial stress states as low as 1 pound per square inch (psi) [2]. Stress states are expected to be higher at the sail suspension points. To determine the effects of radiation dose as a function of stress state, candidate sail materials were loaded in uniaxial tension with stress states ranging from 5,000 psi to 10 psi. Mono-energetic electrons were used to irradiate the sail materials until failure. Results will be presented that indicate the monoenergetic electron dose required to induce material failure as a function of uniaxial tension.

\section{HISTORY}

The concept of using photon pressure for propulsion has been considered since Tsiolkovsky in 1921 [3-8]. In fact, Tsiolkovsky and Tsander wrote of "using tremendous mirrors of very thin sheets" and "using the pressure of sunlight to attain cosmic velocities" in 1924 [3-7]. The term "solar sailing" was coined in the late 1950s and was popularized by Arthur C. Clarke in the short story Sunjammer (The Wind From the Sun) in May 1964 [7]. NASA used sailing techniques to extend the operational life of the Mariner 10 spacecraft in 1974-1975. 


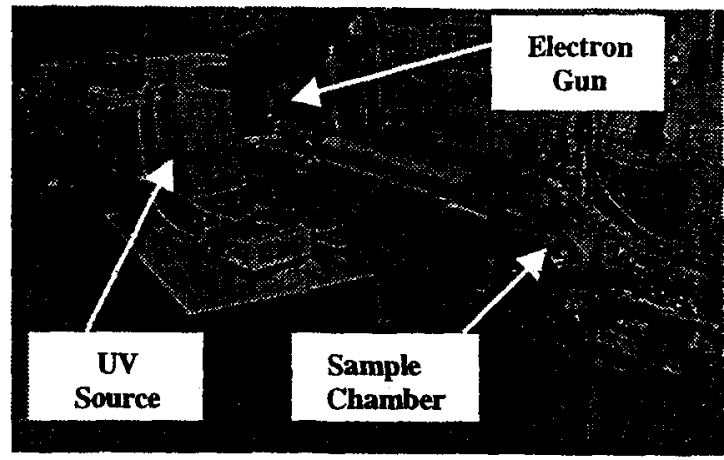

Fig. 1. Low Energy Electron (LEE) System

\section{MATERIAL CANDIDATES}

The materials investigated were $2.0 \times 10^{-6} \mathrm{~m}$ (micron) Mylar with aluminum coating, 2.0 micron Mylar without a coating, and 2.0 micron Teonex with aluminum coating. In an effort to increase the accuracy of the uniaxial stress calculation, thickness measurements were performed on the sail materials. The manufacturer specified thickness of the Mylar samples was 2 microns. Actual thickness measurements of the Mylar film varied from batch to batch. The film thickness ranged from 1.3 microns for the coated Mylar and 1.6 microns for the uncoated Mylar. The manufacturer specified thickness of the Teonex samples was also 2 microns and the measured thickness of these samples was 2.1 microns. The measured thickness values were used to determine the uniaxial stress of the sail material. For simplicity, the sail material will be referred to, in this paper, as having a thickness of 2-microns. Physical characteristics for the solar sail materials investigated are shown in Table 1. Each material was cut into tensile strips prior to mounting in the sample chamber. The materials shown in Table 1 each have an areal density of a few grams per square meter and were selected for this research due to their relevance to solar sail propulsion applications, material availability, and manufacturability. 
A problem in the control system was causing Mariner 10 to go off course. By controlling the attitude of Mariner 10 and the angle of the solar array panels relative to the Sun, ground controllers were able to correct the problem without using precious fuel $[6,8]$.

\section{PHYSICS OF SOLAR SAILS}

A solar sail is a thin membrane material that uses the momentum carried by photons to propel spacecraft. These photons originate from the Sun or can be beamed on to the sail with a laser. If the sail is a good reflector, then the momentum transferred to the sail can be almost doubled. Since the momentum carried by a single photon is extremely small, the surface area of a sail must be large to produce a reasonable acceleration [6], as indicated by:

$$
a=\frac{2 A p_{t}}{m}
$$

where $a$ is the non-relativistic sail acceleration $\left(m / s^{2}\right), A$ is the surface area $\left(\mathrm{m}^{2}\right), \mathrm{p}_{\mathrm{t}}$ is the incident radiation pressure in $\left(\mathrm{N} / \mathrm{m}^{2}\right)$, and $\mathrm{m}$ is the mass $(\mathrm{kg})$. The radiation pressure $\left(\mathrm{p}_{\mathrm{v}}\right)$ varies as the inverse square of the distance from the Sun as shown by:

$$
\mathrm{p}_{\mathrm{t}}=4.56 \times 10^{-6} \frac{(1+\mathrm{R})}{\mathrm{r}_{\mathrm{AU}}^{2}}
$$

where $R$ is the surface reflectivity $(0 \leq R \leq 1)$ of the sail and $r_{A U}$ is the distance to the Sun in astronomical units (AU) [6]. A perfectly reflective solar sail $(R=1)$ at a distance of $1 \mathrm{AU}$ from the Sun experiences a light pressure of $9.1 \mu \mathrm{N} / \mathrm{m}^{2}$. Substituting (2) into (1) for $\mathrm{p}_{1}$ gives the acceleration:

$$
\mathrm{a}=9.12 \times 10^{-6} \frac{(1+\mathrm{R}) A}{\mathrm{mr}_{\mathrm{AU}}^{2}}
$$


Table 1. Physical characteristics of the candidate solar sail materials

\begin{tabular}{|c|c|c|c|}
\hline \multirow[b]{2}{*}{\begin{tabular}{|l} 
Base \\
Polymer \\
Material \\
\end{tabular}} & \multirow[b]{2}{*}{$\begin{array}{l}\text { Polymer } \\
\text { Thickness } \\
\text { (Microns) }\end{array}$} & \multicolumn{2}{|c|}{ Aluminum Coating } \\
\hline & & $\begin{array}{l}\text { Front Surface } \\
\text { Thickness } \\
\text { (nm) }\end{array}$ & $\begin{array}{l}\text { Back Surface } \\
\text { Thickness } \\
\text { (nm) }\end{array}$ \\
\hline Mylar & 1. & $3 \quad 50$ & 0 \\
\hline Mylar & 1. & 6 & 0 \\
\hline Teonex & 2. & 50 & 0 \\
\hline
\end{tabular}

\section{EXPERIMENTAL PROCEDURE}

This paper will describe the results of a total dose to failure investigation. This investigation was designed to determine the electron radiation dose required to induce failure in the sail material. The experimental procedures and results from this test are described below.

\section{Total Dose to Failure Investigation}

In an effort to understand the effects of radiation on specifically tensioned material, appropriately massed weights were suspended from tensile specimens and these tensioned specimens were exposed to $50 \mathrm{keV}$ electrons. The energy of $50 \mathrm{keV}$ was selected because this provided the highest electron energy loss per unit path length, (dE/dx), in the sail material while maintaining a flat, or uniform, dose throughout the material thickness. The goal of this test was to determine the radiation dose that would induce material failure for the specific tension load applied to the candidate sail materials. In the first series of tests, four uncoated Mylar tensile specimens were mounted in the exposure chamber with the following uniaxial stresses: $5000 \mathrm{psi}, 1000 \mathrm{psi}, 100 \mathrm{psi}$, and $10 \mathrm{psi}$. Figure 2 shows a photograph of the samples mounted in the electron beam exposure chamber. The sample tensioned to 5000 psi achieved failure, prior to this photograph, at $5 \times 10^{8}$ rads.

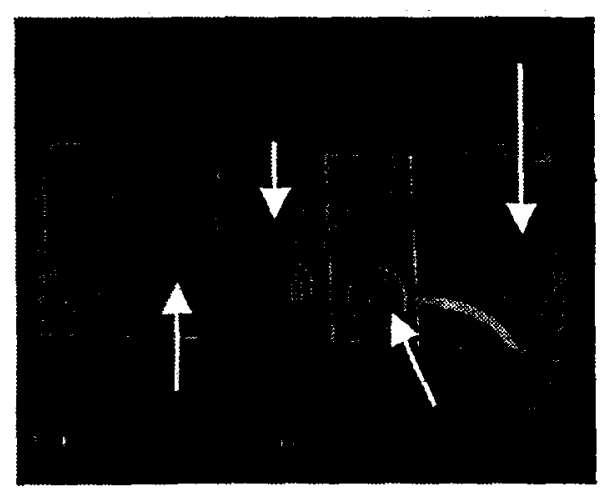

Fig.2. Uncoated Mylar in test chamber with specific uniaxial stress. 
Using (3), a $1000 \mathrm{~kg}$ spacecraft with a perfectly reflective $(R=1)$ sail area of $10^{6} \mathrm{~m}^{2}$ at a distance of 1 AU from the Sun experiences an acceleration of only $1.8 \mathrm{~cm} / \mathrm{s}^{2}$.

Sails are designed to utilize light pressure from the Sun. It is often incorrectly stated that particle pressure from the "solar wind" powers sail spacecraft. Solar wind is composed of low-density protons and electrons moving at high velocity. The pressure due to the solar wind $\left(\mathrm{p}_{\mathrm{w}}\right)$ is on the order of:

$$
p_{w} \sim m_{p} \varphi_{w} v^{2}
$$

where $m_{p}$ is the mass of the proton $(\mathrm{kg}), \varphi_{w}$ is the particle density, and $v$ is the velocity [6]. Near the Earth, a solar wind density of $6 \times 10^{6} \mathrm{~m}^{-3}$ at a velocity of $4 \times 10^{5} \mathrm{~m} / \mathrm{s}$ gives a particle pressure of about $1 \mathrm{nN} / \mathrm{m}^{2}$, which is more than three orders of magnitude smaller than the equivalent photon pressure $[6,9,10]$.

\section{ELECTRON EXPOSURE FACILITIES}

The Environmental Effects Group at Marshall Space Flight Center (MSFC) operates the Low Energy Electron (LEE) test system as shown in Figure 1. It provides the capability to expose materials to a simulated space environment. The selected source of particles for the LEE system is a Kimball Physics EGH-8104A electron gun.

The electron beam energy and emission current can be selected from 1 to $100 \mathrm{keV}$ and 0 to $100 \mu \mathrm{A}$ respectively. Electron flux, from the electron flood gun, is measured using Faraday cups. These cups are located in the test chamber and constantly monitor the electron beam current at the sample position. 
In the second series of tests, two aluminized 2-micron Mylar samples and two aluminized 2-micron Teonex samples were each tensioned to $5000 \mathrm{psi}$. The goal of this test series was to compare the total dose to failure of 2 micron Mylar and 2 micron Teonex subjected to equivalent uniaxial stress loads of $5000 \mathrm{psi}$. In addition, this test would provide statistical data on the total dose to failure of 2-micron Mylar with 5000 psi tensile loads. These materials were exposed to $50 \mathrm{keV}$ electrons. Both test series utilized the method of dose-depth profiling to calculate the absorbed radiation dose in the material as a function of material depth. The method of dose-depth profiling is described in modeling profile section.

\section{Modeling Profile}

The Integrated Tiger Series 3 (ITS3) code was used to determine the dose-depth profile for the mono-energetic electron beams [11]. In order to complete this analysis, specific information is required:

1. Chemical composition and density of each layer,

2 Radiation source type, energy, and orientation from the test sail material, and

3. Number of layers, number of subzones, and the total thickness per layer.

The ITS3 program uses these inputs to generate a library file containing cross-section data for the specific material. The output of the program is a tabular listing of energy deposition and depth for each sail material. The energy deposition was normalized to one source particle. Results from ITS3 were used to convert from incident electron fluence to dose for each specified sail material.

ITS3 divides the material thickness into a number of layers as noted by the "i" subscript. The dose deposited into each layer $\left(D_{i}\right)$ is defined as:

$$
D_{i}=\frac{n_{i} \Delta E_{i}}{m_{i}}
$$


In equation (5), $\mathrm{n}_{\mathrm{i}}$ is the number of electrons impinging on the layer, $\Delta \mathrm{E}_{\mathrm{i}}$ is the energy lost in the layer per electron, and $m_{i}$ is the layer mass. For a homogeneous layer in rectangular geometry, the mass $m_{i}$ is:

$$
m_{i}=\rho_{i} A_{i} t_{i}
$$

where $\rho_{i}$ is the density, $A_{i}$ is the area, and $t_{i}$ is the thickness of the layer.

Substituting equation (6) into (5) gives:

$$
D_{i}=\frac{n_{i} \Delta E_{i}}{\rho_{i} A_{i} t_{i}} .
$$

The energy lost for each electron $\left(\Delta \mathrm{E}_{i}\right)$ is defined as:

$$
\Delta E_{i}=t_{i}\left(\frac{d E}{d x}\right)_{\text {tot }_{i}} \text {, }
$$

where $(\mathrm{dE} / \mathrm{dx})_{\text {tot }}$ is the total energy loss per unit length and $\mathrm{t}_{\mathrm{i}}$ is the thickness of the layer. This expression depends on the energy of the incident electrons and the composition of the target [12].

Electrons interact with the target material primarily by Coulomb scattering from atomic electrons. During the interaction process, electrons can experience potentially large deflections and will follow an erratic path. The range of an electron is defined as the linear distance traveled into a material. The actual path length for an electron could be very different from its calculated range. The total energy loss is the sum of contributions from electron collisions and radiation emission. Photons are emitted from electron bombardment through the production of bremsstrahlung, or “braking” radiation.

Bethe derived expressions for both the collision and radiation energy loss terms for electrons [12]. Since electron energies used in this investigation are reasonably low and non-relativistic, the radiation loss term is much smaller than the collision expression [12]. The resulting expression from equation (8) can be substituted into (7) for $D_{i}$ :

$$
D_{i}=\frac{1}{\rho_{l}} \frac{\eta_{t}}{A t_{i}}\left(\frac{d E}{d x}\right)_{t o t}=\frac{1}{\rho_{l}} \frac{\eta}{A}\left(\frac{d E}{d x}\right)_{t o t}=\frac{1}{\rho_{t}} \phi\left(\frac{d E}{d x}\right)_{t o t}
$$


The ratio $\left(n_{i} / A_{i}\right)$ is defined as the electron fluence $\left(\phi_{i}\right)$ incident on the material layer. The layer mass stopping power $\left(S_{i}\right)$ is defined as:

$$
S_{i}=\frac{1}{\rho_{i}}\left(\frac{d E}{d x}\right)_{\text {tot }_{i}}
$$

Substituting equation (10) into (9) gives the final general expression for layer dose:

$$
D_{i}=S_{i} \phi_{i}
$$

The dose for a given layer is simply the product of the layer mass stopping power and the electron fluence incident to the layer. ITS3 calculates $S_{i}$ with the traditional units of $\mathrm{MeV}-\mathrm{cm}^{2} / \mathrm{g}$. Dose is usually expressed in metric multiples of the rad, and a rad is defined as 100 ergs of deposited energy per unit mass. The layer dose in equation (11) can be written as:

$$
D_{i}(\mathrm{Mrad})=1.6021 \times 10^{-14} S_{i} \phi_{i}
$$

where $S_{\mathrm{i}}$ has units of $\mathrm{MeV}-\mathrm{cm}^{2} / \mathrm{g}$ and $\phi_{\mathrm{i}}$ is in $\mathrm{cm}^{-2}$. The constant $1.6021 \times 10^{-14}$ has units of $\mathrm{Mrad}-\mathrm{g} / \mathrm{MeV}$.

The Teonex and Mylar samples are much thinner than the corresponding range of $50 \mathrm{keV}$ electrons in the material. Therefore, attenuation of electrons is not a consideration. Only the stopping power is unique in each layer.

The electron fluence $(\phi)$ is experimentally measured from raw counts using the Ortec 439 current integrator ( $\left.n_{c}\right)$ as:

$$
\phi=\frac{n_{c}^{\circ} \mathrm{f}}{e A_{f}},
$$

where $f$ is scalar constant set physically on the integrator $\left(10^{-10} \mathrm{C} /\right.$ count $), e$ is the electronic charge $\left(1.6021 \times 10^{-19} \mathrm{C}\right)$, and $A_{f}$ is the cross sectional area of each Faraday cup $\left(0.079 \mathrm{~cm}^{2}\right)$. Substituting equation (13) into (12) and evaluating the known constants, the layer dose is:

$$
\mathrm{D}_{\mathrm{i}}(\mathrm{Mrad})=1.2658 \times 10^{-4} \mathrm{n}_{\mathrm{c}} \mathrm{S}_{\mathrm{i}}
$$

The constant $1.2658 \times 10^{-4}$ has units of $(\mathrm{Mrad}-\mathrm{g}) /\left(\mathrm{MeV}-\mathrm{cm}^{2}\right)$. Equation (14) can be used to generate a dose-depth profile for each layer in the ITS3 analysis. The calculated electron dose for the front and rear surfaces are averaged together to give an estimate of the total dose. Averaging can be used in this situation because the dose-depth profile for both samples is flat with little variance. Figure 3 shows the flat dose-depth profile for $50 \mathrm{keV}$ electrons in Mylar. 


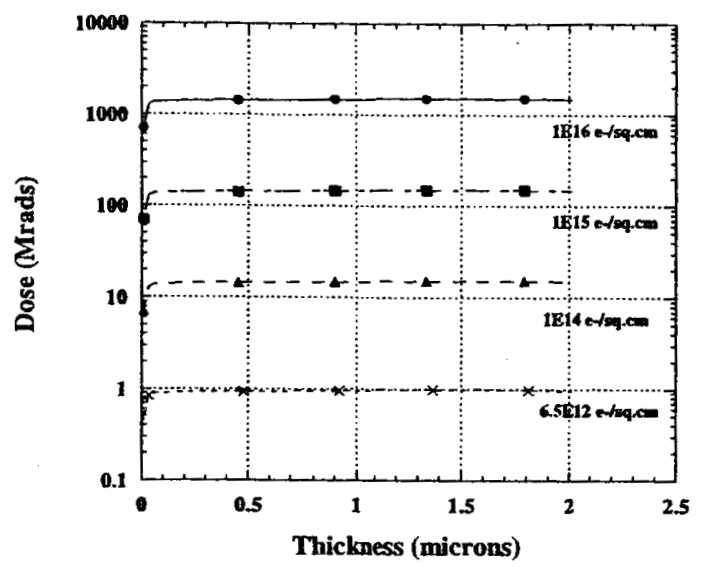

Fig. 3 Uniform or "Flat" electron radiation dose as a function of Mylar thickness

\section{RESULTS}

Results from the initial series of investigations can be found in Table 2.

Table 2. Results of the sail irradiation tests

\begin{tabular}{|c|c|c|c|c|}
\hline Material & $\begin{array}{l}\text { Polymer } \\
\text { Thickness } \\
\text { (Microns) } \\
\end{array}$ & \begin{tabular}{|l|} 
Maximum Tested \\
Total Dose (Mrad)
\end{tabular} & $\begin{array}{l}\text { Initial Stress } \\
\text { (psi) }\end{array}$ & $\begin{array}{l}\text { Failure } \\
\text { Observed ? }\end{array}$ \\
\hline Mylar & 1.6 & \begin{tabular}{|l|} 
\\
500
\end{tabular} & 5,000 & Yes \\
\hline Mylar & 1.6 & 16.740 & 1,000 & No \\
\hline Mylar & 1.6 & 16,740 & 100 & No \\
\hline Mylar & 1.6 & 16,740 & 10 & No \\
\hline Al/Mylar & 1.3 & 4,900 & 5,000 & Yes \\
\hline Al/Mylar & 1.3 & 22,840 & 5,000 & No \\
\hline Al/Teonex & 2.1 & 22,840 & 5,000 & No \\
\hline Al/Teonex & 2.1 & 22,840 & 5,000 & No \\
\hline
\end{tabular}




\section{Total Dose to Failure Investigation}

In the first series of tests, the uncoated Mylar sample, tensioned to 5,000 psi, achieved failure at an absorbed dose level of $5 \times 10^{8} \mathrm{rad}$ ( $500 \mathrm{Mrad}$ ). The samples tensioned with $1000 \mathrm{psi}, 100 \mathrm{psi}$, and $10 \mathrm{psi}$ did not achieve failure prior to the termination of the test. When the test concluded, the remaining Mylar sail materials received 16.74 Grads. The 100 psi and 10 psi samples were experiencing substantial charging effects, which was significant in the decision to terminate the exposure prior to material failure.

In the second series of tests, one of the aluminized Mylar samples achieved failure at $4.9 \times 10^{9} \mathrm{rad}(4900 \mathrm{Mrad})$. The remaining samples received $2.284 \times 10^{10} \mathrm{rad}(22,840 \mathrm{Mrad})$ and did not fail.

It should be noted that once the electron exposure was terminated and the test chamber was re-pressurized to ambient pressure, the samples were removed for further study. During the removal process, the sail material was observed to be extremely brittle and fragile. These qualitative indications led the authors to note that electron exposure did have a significant, potentially degrading, effect on the material. It should also be noted that control, unexposed, samples of Mylar and Teonex with equivalent uniaxial stress did not experience failure after 90 days. These samples were subjected to equivalent loads as those samples that were exposed to electron radiation in vacuum. These control samples were suspended in the laboratory, in a controlled ambient laboratory environment. Figure 4 shows the relationship between stress state and radiation dosage required for material failure.

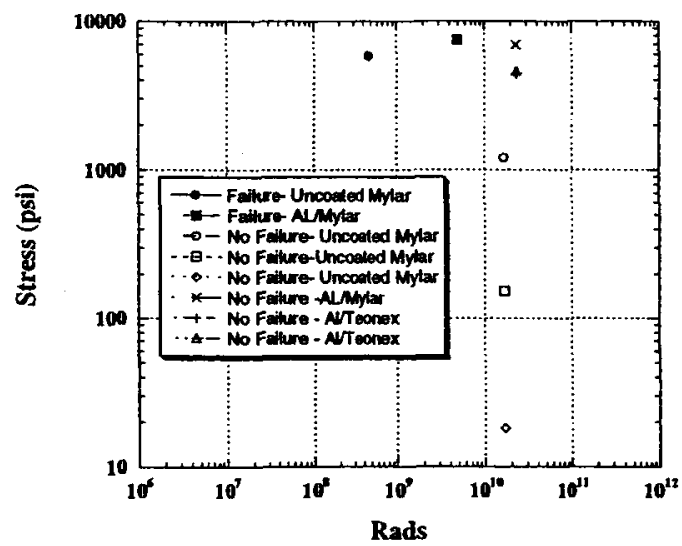

Fig. 4 Relationship between tension load and radiation dosage in aluminised Mylar and Teonex sail materials 


\section{Functional Engineering Lifetimes}

Determination of the functional engineering lifetime of candidate sail material is based on the predicted radiation dose a sail would receive in a specific orbital environment. Accurate dose predictions require an accurate orbital environment definition. The data presented below was generated using the best orbital environment definitions available at this time. Efforts are underway to improve the accuracy of these environment definition models and the author cautions using this as baseline data until the improvements are completed in the environment definition models.

Figure 5 shows a dose-depth profile for 2.5 micron Mylar in a Geosynchronous Transfer Orbit (GTO). This dose profile is representative of the radiation dose a sail material would receive in a GTO environment for 1 year.

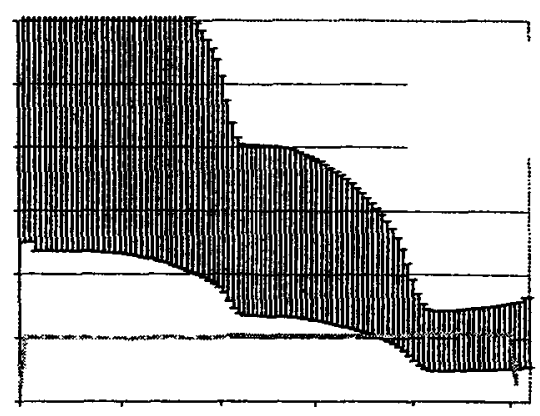

Fig. 5. Dose-Depth profile for 2.5 micron Mylar in a GTO environment for 1 year.

At present, several solar sail missions are baselined to operate at the Sun-Earth LaGrange point number 1 (L1). This is a location is space approximately 1 million $\mathrm{Km}$ from Earth, in a line between the Earth and Sun. Radiation environments are currently being defined for this location in space. Very preliminary data was obtained to generate a dose-depth profile to indicate the radiation dose a sail would receive during a 1 year at L1. Figure 6 shows the dosedepth profile of a Mylar sail material at L1 for 1 year. 


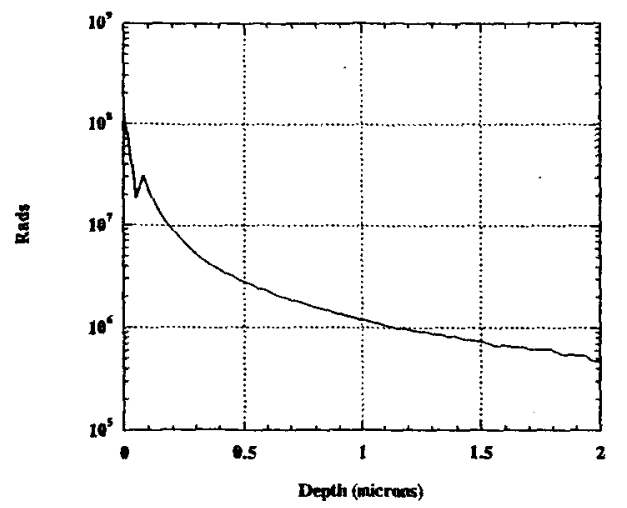

Fig. 6. Dose-Depth profile for 2.0 micron Mylar in an L1 environment for 1 year

Figures 5 and 6 clearly indicate that radiation dose varies with orbital environment. Likewise, the functional engineering lifetime of the sail will vary with orbital environment. Although quantitative values of engineering functional lifetimes cannot be accurately assessed from these preliminary results, this data and the dose-depth profiles begin to provide some insight into the approximate timeframe of sail functional service. The data reported as a result of this investigation tends to indicate that sail materials, with minimal applied stress can possibly perform their engineering function for a multi-year mission in environments comparable to those shown in figure 6. Before accurate lifetimes can be assessed, enhanced environment definitions must be developed and combined effects material exposures with appropriate stress states must be performed

\section{CONCLUSIONS}

Once thought to be difficult or impossible, solar sailing has come out of science fiction and into the realm of possibility. Practical sails must be resistant to the effects of long duration space environment exposure. Results from this research indicate that the candidate sail materials, Mylar and teonex, can survive significant doses of electron radiation while under high uniaxial stress. A method to quantify this degradation, relative to the functional engineering lifetime of the sail material, was introduced.

Enhanced space environment definitions and prediction models are needed to determine precise engineering functional lifetimes for solar sail materials. 
The limited, preliminary data indicates that survivable solar sail missions are, at least, possible from a materials survivability standpoint. As more data becomes available, more confidence in the duration of solar sail missions will be achieved.

\section{REFERENCES}

1. Edwards, D., Hubbs, W., Stanaland, T., Hollerman, A., and Altstatt, R., 2002, "Characterization of Space Environmental Effects on Candidate Solar Sail Material", Proceedings, 47th International Symposium on Optical Science and Technology, Seattle, WA.

2. Greschik, G. and Mikulas, M.M. “Design Study of a Square Solar Sail Architecture”, Journal of Spacecraft and Rockets, Vol. 39, No. 5, September-October, 2002.

3. Tsander, F.A., 1969, "From a Scientific Heritage", Translation, pp. 1-92, NASA TT F-541, National Aeronautics and Space Administration, Washington, DC.

4. Blagonravov, A.A., Editor, 1968, "K.E. Tsiolkovsky Selected Works", Translation by G. Yankovsky, pp. 140-163, Mir Publishers, Moscow, USSR.

5. Tsander, F.A., 1964, "The Use of Light Pressure for Flight in Interplanetary Space, Problems of Flight by Jet Propulsion", L.K. Korneev, Editor, pp. 303-321, Israel Program for Scientific Translations, Jerusalem, Israel.

6. McInnes, C.R., 1999, "Solar Sailing: Technology, Dynamics and Mission Applications", pp. 1-50, Praxis Publishing, Chichester, United Kingdom.

7. Clarke, A.C., 1990, "The Wind From the Sun", A.C. Clarke, Editor, "Project Solar Sail", pp.9-31, Penguin Books, New York, NY.

8. Souza, D.M., 1994, "Space Sailing", pp. 1-63, Lerner Publications Company, Minneapolis, MN. 
9. "NASA Marshall Space Flight Center Solar Wind", NASA Marshall Space Flight Center, MSFC, AL, http://science.msfc.nasa.gov/ss//pad/solar/sun wind.htm/.

10. "Science news and information about the Sun-Earth space environment", http://www.spaceweather.com/.

11. Halbleib, J.A., Kensek, R.P., Valdez, G.D., Seltzer, S.M., and Berger, M.J., 1992, "ITS Version 3.0: The Integrated Tiger Series of Coupled Electron/Photon Monte Carlo Transport Codes", SAN91-1634, Sandia National Laboratories, Albuquerque, NM.

12. Krane, K.S., 1988, "Introductory Nuclear Physics", pp. 196-198, John Wiley and Sons Publishing, New York, NY.

13. Hollerman, W.A., et al., 2003, "Accelerator-Based PIXE and STIM Analysis of Candidate Solar Sail Materials", Proceedings of the Seventeenth International Conference on the Application of Accelerators in Research and Industry, Edited by J. Duggan and I. Morgan, American Institute of Physics, Denton, TX.

14. Cember, H., 1983, "Introduction to Health Physics, Second Edition”, pp. 99-101, Pergamon Press Publications, New York, NY.

15. McCrum, N.G., Buckley, C.P., and Bucknall, C.B., 1988, "Principles of Polymer Engineering”, pp. 101-166, Oxford Science Publications, New York, NY. 